\title{
The n-Hosoya Polynomial of $W_{\alpha} \otimes C_{\beta}$
}

\section{Ahmed M. Ali \\ ahmedgraph@uomosul.edu.iq \\ College of Computer Sciences and Mathematics \\ University of Mosul}

Received on: 21/08/2011

\author{
Haveen G. Ahmed \\ haveenagali@gmail.com \\ College of Science \\ University of Dohuk
}

Accepted on: 19/04/2012

\begin{abstract}
For a wheel $\mathrm{W}_{\alpha}$ and a cycle $\mathrm{C}_{\beta}$ the composite graphs $\mathrm{W}_{\alpha} \otimes \mathrm{C}_{\beta}$ is constructed from the union of $\mathrm{W}_{\alpha}$ and $\mathrm{C}_{\beta}$ and adding the edges $\mathrm{u}_{1} \mathrm{u}_{2}, \mathrm{u}_{1} \mathrm{v}_{2}, \mathrm{v}_{1} \mathrm{u}_{2}$ and $\mathrm{v}_{1} \mathrm{v}_{2}$, where $\mathrm{u}_{1} \mathrm{v}_{1}$ is an edge of $\mathrm{W}_{\alpha}$ and $\mathrm{u}_{2} \mathrm{v}_{2}$ is an edge of $\mathrm{C}_{\beta}$. The $\mathrm{n}$ - diameter, the $\mathrm{n}$-Hosoya polynomial and the $n-$ Wiener index of $W_{\alpha} \otimes C_{\beta}$ are obtained in this paper.

Keywords: n-distance, n-Hosoya polynomial, n-Wiener index, Wheel and cycle.

$$
\begin{aligned}
& \text { متعددة حدود هوسويا - n للبيان } \\
& \text { هافين جلال أحمد } \\
& \text { كلية العلوم } \\
& \text { جامعة دهوك كله } \\
& \text { تاريخ قبول البحث: 2012/04/19 } \\
& \text { كلية علوم الحاسوب والرياضيات محمد علي } \\
& \text { تاريخ استلام البحث: 2011/08/21 }
\end{aligned}
$$

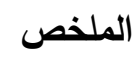

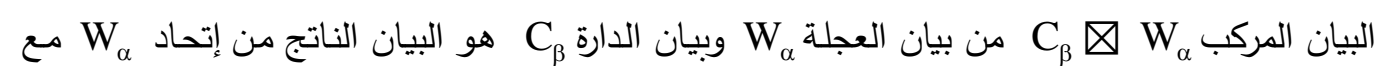

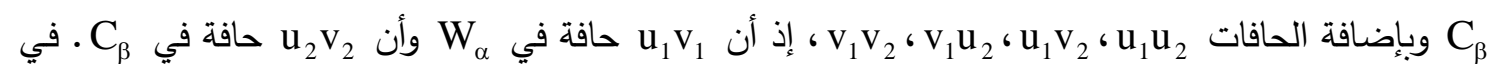

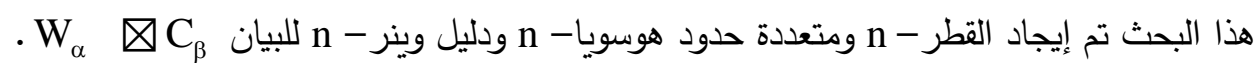

$$
\begin{aligned}
& \text { الكلمات المفتاحية : مسافة -n ، متعددة حدود هوسويا -n ، دليل وينر -n ، العجلة والدارة. }
\end{aligned}
$$

\section{Introduction:}

For the definitions of concepts and notations used in this paper, see the references [6, 7 and 8]. Some authors defined the minimum distance between two nonempty subsets of vertices of a connected graph $G$ by [7]: $\mathrm{d}_{\text {min }}(\mathrm{A}, \mathrm{B})=\min \{\mathrm{d}(\mathrm{a}, \mathrm{b}): \mathrm{a} \in \mathrm{A}, \mathrm{b} \in \mathrm{B}\}$, where $A$ and $B$ are nonempty subsets of vertices of a connected graph $G$.

The n-distance in a connected graph $\mathrm{G}=(\mathrm{V}, \mathrm{E})[4]$ is the minimum distance from a singleton vertex $\mathrm{v} \in \mathrm{V}$ to an (n-1)-subset $\mathrm{S}, \mathrm{S} \subseteq \mathrm{V}$, that is $d_{n}(v, S)=\min \{d(v, u): u \in S\}, 2 \leq n \leq p$, in which $p$ is the order of $G$. 
It is clear that :

$\mathrm{d}_{\mathrm{n}}(\mathrm{v}, \mathrm{S})=0$; when $\mathrm{v} \in \mathrm{S}$,

$\mathrm{d}_{\mathrm{n}}(\mathrm{v}, \mathrm{S}) \geq 1$; when $\mathrm{v} \notin \mathrm{S}$.

When $\mathrm{n}=2$, we get the (ordinary) distance $\mathrm{d}(\mathrm{u}, \mathrm{v})$.

The $n$-diameter of $\mathrm{G}$ is defined by

$\delta_{\mathrm{n}}=\delta_{\mathrm{n}}(\mathrm{G})=\max \left\{\mathrm{d}_{\mathrm{n}}(\mathrm{v}, \mathrm{S}): \mathrm{v} \in \mathrm{V}(\mathrm{G}),|\mathrm{S}|=\mathrm{n}-1, \mathrm{~S} \subseteq \mathrm{V}(\mathrm{G})\right\}$.

The $n$-Hosoya polynomial of $G$ of order $p$ is defined by

$$
\mathrm{H}_{\mathrm{n}}(\mathrm{G} ; \mathrm{x})=\sum_{\mathrm{k}=1}^{\delta_{\mathrm{n}}} \mathrm{C}_{\mathrm{n}}(\mathrm{G}, \mathrm{k}) \mathrm{x}^{\mathrm{k}} \text {, }
$$

where $C_{n}(G, k)$ is the number of order pairs $(v, S), v \in V(G), S \subseteq V(G),|S|=n-1$, such that $\mathrm{d}_{\mathrm{n}}(\mathrm{v}, \mathrm{S})=\mathrm{k}, 2 \leq \mathrm{n} \leq \mathrm{p}$.

One can easily show that [4].

$C_{n}(G, 1)=p\left(\begin{array}{l}p-1 \\ n-1\end{array}\right)-\sum_{v \in V(G)}\left(\begin{array}{c}p-1-\operatorname{deg} v \\ n-1\end{array}\right)$.

The n-Hosoya polynomial of a vertex $v$ in $G$, denoted by $H_{n}(v, G$; $x)$, is defined [4] by

$$
\mathrm{H}_{\mathrm{n}}(\mathrm{v}, \mathrm{G} ; \mathrm{x})=\sum_{\mathrm{k} \geq 1} \mathrm{C}_{\mathrm{n}}(\mathrm{v}, \mathrm{G}, \mathrm{k}) \mathrm{x}^{\mathrm{k}} \text {, }
$$

where $C_{n}(v, G, k)$ is the number of $(n-1)$-subsets of vertices $S$ such that $d_{n}(v, S)=k$. It is clear that

$$
\mathrm{C}_{\mathrm{n}}(\mathrm{G}, \mathrm{k})=\sum_{\mathrm{v} \in \mathrm{V}(\mathrm{G})} \mathrm{C}_{\mathrm{n}}(\mathrm{v}, \mathrm{G}, \mathrm{k}), \text { for } 1 \leq \mathrm{k} \leq \delta_{\mathrm{n}}
$$

and

$$
\mathrm{H}_{\mathrm{n}}(\mathrm{G} ; \mathrm{x})=\sum_{\mathrm{v} \in \mathrm{V}(\mathrm{G})} \mathrm{H}_{\mathrm{n}}(\mathrm{v}, \mathrm{G} ; \mathrm{x}) \text {, }
$$

The $n$-Wiener index of $\mathrm{G}$ is defined by

$$
\mathrm{W}_{\mathrm{n}}(\mathrm{G})=\left.\frac{\mathrm{d}}{\mathrm{dx}} \mathrm{H}_{\mathrm{n}}(\mathrm{G} ; \mathrm{x})\right|_{\mathrm{x}=1}=\sum_{\mathrm{k}=1}^{\delta_{\mathrm{n}}} \mathrm{kC}_{\mathrm{n}}(\mathrm{G}, \mathrm{k}) \text {. }
$$

In [2], H. G. Ahmed gave the following result :

Lemma: Let $v$ be any vertex of a connected graph $G$. If there are $t_{1}$ vertices of distance $\mathrm{k} \geq 1$ from $\mathrm{v}$, and there are $\mathrm{t}_{2}$ vertices of distance more than $\mathrm{k}$ from $\mathrm{v}$, then

$$
\mathrm{C}_{\mathrm{n}}(\mathrm{v}, \mathrm{G}, \mathrm{k})=\left(\begin{array}{c}
\mathrm{t}_{1}+\mathrm{t}_{2} \\
\mathrm{n}-1
\end{array}\right)-\left(\begin{array}{c}
\mathrm{t}_{2} \\
\mathrm{n}-1
\end{array}\right) . \#
$$

\section{Definition(1): [1]}

Let $G_{1}$ and $G_{2}$ be disjoint connected graphs, and let $u_{1} v_{1}$ be an edge of $G_{1}$ and $u_{2} v_{2}$ be an edge of $G_{2}$, then the composite graph $G_{1} \otimes G_{2}$ is the graph constructed from $\mathrm{G}_{1}$ and $\mathrm{G}_{2}$ by adding the edges $\mathrm{u}_{1} \mathrm{u}_{2}, \mathrm{u}_{1} \mathrm{v}_{2}, \mathrm{v}_{1} \mathrm{u}_{2}$ and $\mathrm{v}_{1} \mathrm{v}_{2}$. It is clear that $\mathrm{p}\left(\mathrm{G}_{1} \otimes \mathrm{G}_{2}\right)=\mathrm{p}\left(\mathrm{G}_{1}\right)+\mathrm{p}\left(\mathrm{G}_{2}\right)$ and $\mathrm{q}\left(\mathrm{G}_{1} \otimes \mathrm{G}_{2}\right)=\mathrm{q}\left(\mathrm{G}_{1}\right)+\mathrm{q}\left(\mathrm{G}_{2}\right)+4$.

To simplify our discussion, we give the following : 


\section{Definition(2):}

For every vertex $\mathrm{v}$ of a connected graph $\mathrm{G}$ and each $\mathrm{k}, 1 \leq \mathrm{k} \leq \delta_{\mathrm{n}}$, we define $: \mathrm{N}_{\mathrm{k}}^{\mathrm{e}}(\mathrm{v})$ is the number of vertices $\mathrm{w}$ of $\mathrm{G}$ such that $\mathrm{d}(\mathrm{v}, \mathrm{w})=\mathrm{k}$, and $\mathrm{N}_{\mathrm{k}}^{+}(\mathrm{v})$ is the number of vertices $w$ of $G$ such that $d(v, w)>k$.

Finally, it seems to us that it is impossible to obtain the $\mathrm{n}$ - Hosoya polynomial of $G_{1} \otimes G_{2}$ for any disjoint connected graphs $G_{1}$ and $G_{2}$ in terms of $H_{n}\left(G_{1} ; x\right)$ and $H_{n}\left(G_{2} ; x\right)$.Therefore, in [5], A. M. Ali obtained $H_{n}\left(G_{1} \otimes G_{2} ; x\right)$ where $G_{1}$ is a complete graph and $\mathrm{G}_{2}$ is a special graph such as a complete graph, a complete bipartite, a wheel, or a cycle and in [3]; H.G. Ahmed obtained $H_{n}\left(G_{1} \otimes G_{2} ; x\right)$ where $G_{1}$ and $G_{2}$ are wheels $W_{\alpha}$ and $W_{\beta}$. In the continuation of such work, we take $G_{1}$ as a wheel $\mathrm{W}_{\alpha}$ and $\mathrm{G}_{2}$ as a cycle $\mathrm{C}_{\beta}$ and find the $\mathrm{n}$ - diameter, $\mathrm{n}$ - Hosoya polynomial and $\mathrm{n}-$ Wiener index of $\mathrm{W}_{\alpha} \otimes \mathrm{C}_{\beta}$.

\section{The n-Hosoya Polynomial of $\mathrm{W}_{\alpha} \otimes \mathrm{C}_{\beta}$ :}

The graph $G=W_{\alpha} \otimes C_{\beta}$, for $\alpha \geq 6$ and $\beta \geq 4$ is shown in Fig. 2.1. It is clear that $\mathrm{p}(\mathrm{G})=\alpha+\beta, \mathrm{q}(\mathrm{G})=2 \alpha+\beta+2$ and $\operatorname{diamG}=\left\lfloor\frac{\beta-1}{2}\right\rfloor+3$.

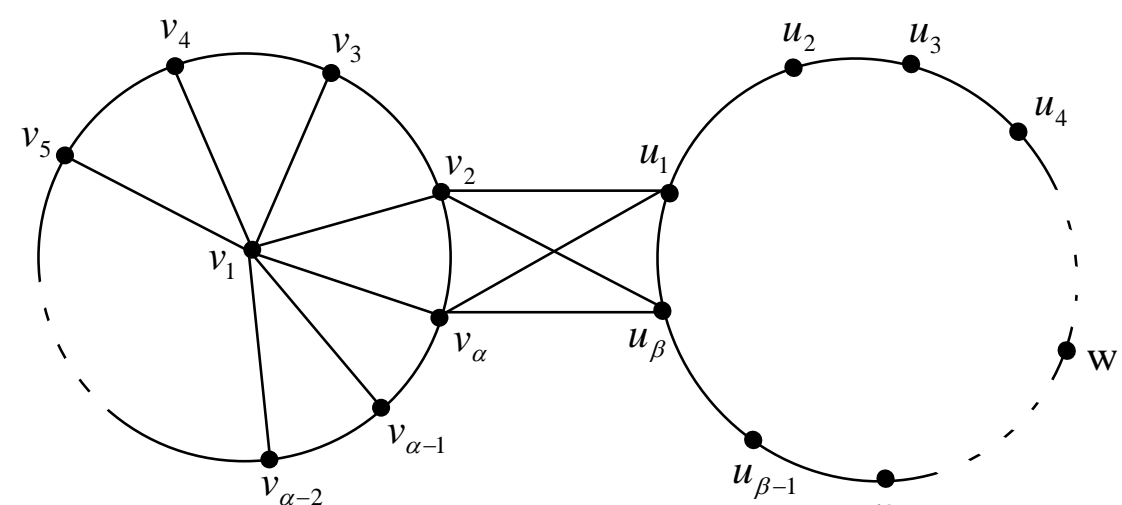

Fig. 2.1. The graph $G=W_{\alpha} \otimes C_{\beta}, \alpha \geq 6$ and $\beta-\beta \geq 4$

Let $\mathrm{V}=\mathrm{V}\left(\mathrm{W}_{\alpha}\right)=\left\{\mathrm{v}_{1}, \mathrm{v}_{2}, \ldots, \mathrm{v}_{\alpha}\right\}$ and $\mathrm{U}=\mathrm{V}\left(\mathrm{C}_{\beta}\right)=\left\{\mathrm{u}_{1}, \mathrm{u}_{2}, \ldots, \mathrm{u}_{\beta}\right\}$.

The following proposition determines the n-diameter of $\mathrm{G}=\mathrm{W}_{\alpha} \otimes \mathrm{C}_{\beta}$.

Proposition 2.1: For $2 \leq \mathrm{n} \leq \mathrm{p}(=\alpha+\beta), \alpha \geq 6$ and $\beta \geq 4$, we have

$$
\operatorname{diam}_{n}(G)=\left[\begin{array}{ll}
\left\lfloor\frac{\beta-1}{2}\right\rfloor+3, & \text { for } 2 \leq n \leq \alpha-4, \\
\left\lfloor\frac{\beta-1}{2}\right\rfloor+2, & \text { for } \alpha-3 \leq n \leq \alpha-1, \\
\left\lfloor\frac{\beta-1}{2}\right\rfloor+1, & \text { for } \alpha \leq n \leq \alpha+1, \\
\left\lfloor\frac{\alpha+\beta-n}{2}\right\rfloor+1, & \text { for } \alpha+2 \leq n \leq \alpha+\beta .
\end{array}\right.
$$


Proof: For $2 \leq \mathrm{n} \leq \alpha-4$, we take $\mathrm{S} \subseteq\left\{\mathrm{v}_{4}, \mathrm{v}_{5}, \ldots, \mathrm{v}_{\alpha-2}\right\}$ and $\mathrm{w}=\mathrm{u}_{\beta / 2}\left(\right.$ or $\left.\mathrm{w}=\mathrm{u}_{\beta / 2+1}\right)$ for even $\beta$ and $w=u_{(\beta+1) / 2}$ for odd $\beta$. Then, $d_{n}(w, S)=\left\lfloor\frac{\beta-1}{2}\right\rfloor+3$ which is max. of such values of $n$.

For $\alpha-3 \leq \mathrm{n} \leq \alpha-1$, we take $\mathrm{S} \subseteq\left\{\mathrm{v}_{1}, \mathrm{v}_{3}, \mathrm{v}_{4}, \ldots, \mathrm{v}_{\alpha-1}\right\}$ with $|\mathrm{S}|=\mathrm{n}-1$, then $\mathrm{d}_{\mathrm{n}}(\mathrm{w}, \mathrm{S})=\left\lfloor\frac{\beta-1}{2}\right\rfloor+2=\operatorname{diam}_{\mathrm{n}} \mathrm{G}$.

Similarly, for $\alpha \leq \mathrm{n} \leq \alpha+1$, we take $|\mathrm{S}|=\mathrm{n}-1$ and $\mathrm{S} \subseteq \mathrm{V}\left(\mathrm{W}_{\alpha}\right)$, then $d_{n}(w, S)=\left\lfloor\frac{\beta-1}{2}\right\rfloor+1=\operatorname{diam}_{n} G$. Finally, for $\alpha+2 \leq n \leq \alpha+\beta$, we have $2 \leq n-\alpha \leq \beta$ $\operatorname{diam}_{n}(G)=\operatorname{diam}_{n-\alpha}\left(C_{\beta}\right)=\left\lfloor\frac{\beta-(n-\alpha)}{2}\right\rfloor+1=\left\lfloor\frac{\alpha+\beta-n}{2}\right\rfloor+1$. In this case, we take $S$ containing $\mathrm{V}\left(\mathrm{W}_{\alpha}\right)$ with the sequence of vertices $\mathrm{u}_{1}, \mathrm{u}_{\beta}, \mathrm{u}_{2}, \mathrm{u}_{\beta-1}, \ldots$ and so on to $\beta-1$ of vertices of $\mathrm{V}\left(\mathrm{C}_{\beta}\right)$.\#

Since $H_{n}(G ; x)=\sum_{k=1}^{\delta_{n}} C_{n}(G, k) x^{k}$, where $\delta_{n}$ is the $n$-diameter of $G$ determined by Proposition 2.1, we shall find $C_{n}(G, k), 1 \leq k \leq \delta_{n}$ in order to get $H_{n}(G ; x)$.

Using (1.3), we get

$$
\mathrm{C}_{\mathrm{n}}(\mathrm{G}, 1)=\mathrm{p}\left(\begin{array}{l}
\mathrm{p}-1 \\
\mathrm{n}-1
\end{array}\right)-(\alpha-3)\left(\begin{array}{l}
\mathrm{p}-4 \\
\mathrm{n}-1
\end{array}\right)-(\beta-2)\left(\begin{array}{l}
\mathrm{p}-3 \\
\mathrm{n}-1
\end{array}\right)-2\left(\begin{array}{l}
\mathrm{p}-5 \\
\mathrm{n}-1
\end{array}\right)-2\left(\begin{array}{l}
\mathrm{p}-6 \\
\mathrm{n}-1
\end{array}\right)-\left(\begin{array}{c}
\beta \\
\mathrm{n}-1
\end{array}\right)
$$

Proposition 2.2: For $2 \leq \mathrm{n} \leq \mathrm{p}$ and $\alpha \geq 6, \beta \geq 4$, we have

$$
\begin{array}{r}
\mathrm{C}_{\mathrm{n}}(\mathrm{G}, 2)=(\beta-2)\left(\begin{array}{l}
\mathrm{p}-3 \\
\mathrm{n}-1
\end{array}\right)+(\alpha-3)\left(\begin{array}{l}
\mathrm{p}-4 \\
\mathrm{n}-1
\end{array}\right)+2\left(\begin{array}{l}
\mathrm{p}-6 \\
\mathrm{n}-1
\end{array}\right)-(\alpha-6)\left(\begin{array}{l}
\beta \\
\mathrm{n}-1
\end{array}\right)-3\left(\begin{array}{l}
\beta-2 \\
\mathrm{n}-1
\end{array}\right) \\
-2\left(\begin{array}{l}
\beta-4 \\
\mathrm{n}-1
\end{array}\right)-(\beta-6)\left(\begin{array}{l}
\mathrm{p}-5 \\
\mathrm{n}-1
\end{array}\right)-2\left(\begin{array}{l}
\mathrm{p}-7 \\
\mathrm{n}-1
\end{array}\right)-2\left(\begin{array}{l}
\mathrm{p}-10 \\
\mathrm{n}-1
\end{array}\right)
\end{array}
$$

Proof: From Fig. 2.1, it is clear that $\mathrm{N}_{2}^{=}\left(\mathrm{v}_{1}\right)=2$ and $\mathrm{N}_{2}^{+}\left(\mathrm{v}_{1}\right)=\beta-2$. Thus, by (1.7)

$\mathrm{C}_{\mathrm{n}}\left(\mathrm{v}_{1}, \mathrm{G}, 2\right)=\left(\begin{array}{c}\beta \\ \mathrm{n}-1\end{array}\right)-\left(\begin{array}{c}\beta-2 \\ \mathrm{n}-1\end{array}\right)$.

$\mathrm{N}_{2}^{=}\left(\mathrm{v}_{2}\right)=\alpha-2$ and $\mathrm{N}_{2}^{+}\left(\mathrm{v}_{2}\right)=\beta-4$. Thus, by (1.7)

$\mathrm{C}_{\mathrm{n}}\left(\mathrm{v}_{2}, \mathrm{G}, 2\right)=\mathrm{C}_{\mathrm{n}}\left(\mathrm{v}_{\alpha}, \mathrm{G}, 2\right)=\left(\begin{array}{c}\mathrm{p}-6 \\ \mathrm{n}-1\end{array}\right)-\left(\begin{array}{c}\beta-4 \\ \mathrm{n}-1\end{array}\right)$.

$\mathrm{N}_{2}^{=}\left(\mathrm{v}_{3}\right)=\alpha-2$ and $\mathrm{N}_{2}^{+}\left(\mathrm{v}_{2}\right)=\beta-2$. Thus, by (1.7)

$\mathrm{C}_{\mathrm{n}}\left(\mathrm{v}_{3}, \mathrm{G}, 2\right)=\mathrm{C}_{\mathrm{n}}\left(\mathrm{v}_{\alpha-1}, \mathrm{G}, 2\right)=\left(\begin{array}{c}\mathrm{p}-4 \\ \mathrm{n}-1\end{array}\right)-\left(\begin{array}{c}\beta-2 \\ \mathrm{n}-1\end{array}\right)$.

For $\mathrm{i}=4,5, \ldots, \alpha-2$, then $\mathrm{N}_{2}^{=}\left(\mathrm{v}_{\mathrm{i}}\right)=\alpha-4$ and $\mathrm{N}_{2}^{+}\left(\mathrm{v}_{\mathrm{i}}\right)=\beta$. Thus, by (1.7)

$\mathrm{C}_{\mathrm{n}}\left(\mathrm{v}_{\mathrm{i}}, \mathrm{G}, 2\right)=\left(\begin{array}{l}\mathrm{p}-4 \\ \mathrm{n}-1\end{array}\right)-\left(\begin{array}{c}\beta \\ \mathrm{n}-1\end{array}\right)$.

From (2.3) - (2.6) we obtain 
$C_{n}(V, G, 2)=(\alpha-3)\left(\begin{array}{l}p-4 \\ n-1\end{array}\right)+2\left(\begin{array}{l}p-6 \\ n-1\end{array}\right)-(\alpha-6)\left(\begin{array}{c}\beta \\ n-1\end{array}\right)-3\left(\begin{array}{l}\beta-2 \\ n-1\end{array}\right)-2\left(\begin{array}{l}\beta-4 \\ n-1\end{array}\right)$.

Now, we find $\mathrm{C}_{\mathrm{n}}(\mathrm{U}, \mathrm{G}, 2)$.

$\mathrm{N}_{2}^{=}\left(\mathrm{u}_{1}\right)=5$ and $\mathrm{N}_{2}^{+}\left(\mathrm{u}_{1}\right)=\mathrm{p}-10$. Thus, by (1.7)

$\mathrm{C}_{\mathrm{n}}\left(\mathrm{u}_{1}, \mathrm{G}, 2\right)=\mathrm{C}_{\mathrm{n}}\left(\mathrm{u}_{\beta}, \mathrm{G}, 2\right)=\left(\begin{array}{c}\mathrm{p}-5 \\ \mathrm{n}-1\end{array}\right)-\left(\begin{array}{c}\mathrm{p}-10 \\ \mathrm{n}-1\end{array}\right)$.

$\mathrm{N}_{2}^{=}\left(\mathrm{u}_{2}\right)=4$ and $\mathrm{N}_{2}^{+}\left(\mathrm{u}_{2}\right)=\mathrm{p}-7$. Thus, by $(1.7)$

$\mathrm{C}_{\mathrm{n}}\left(\mathrm{u}_{2}, \mathrm{G}, 2\right)=\mathrm{C}_{\mathrm{n}}\left(\mathrm{u}_{\beta-1}, \mathrm{G}, 2\right)=\left(\begin{array}{c}\mathrm{p}-3 \\ \mathrm{n}-1\end{array}\right)-\left(\begin{array}{l}\mathrm{p}-7 \\ \mathrm{n}-1\end{array}\right)$.

For $\mathrm{u}_{\mathrm{i}}, \mathrm{i}=3,4, \ldots, \beta-2$, then $\mathrm{N}_{2}^{=}\left(\mathrm{u}_{\mathrm{i}}\right)=2$ and $\mathrm{N}_{2}^{+}\left(\mathrm{u}_{\mathrm{i}}\right)=\mathrm{p}-5$. Thus , by (1.7)

$\mathrm{C}_{\mathrm{n}}\left(\mathrm{u}_{\mathrm{i}}, \mathrm{G}, 2\right)=\left(\begin{array}{l}\mathrm{p}-3 \\ \mathrm{n}-1\end{array}\right)-\left(\begin{array}{c}\mathrm{p}-5 \\ \mathrm{n}-1\end{array}\right), \mathrm{i}=3,4, \ldots, \beta-2$.

From (2.8), (2.9), and (2.10) we get

$C_{n}(U, G, 2)=(\beta-2)\left(\begin{array}{l}p-3 \\ n-1\end{array}\right)-(\beta-6)\left(\begin{array}{l}p-5 \\ n-1\end{array}\right)-2\left(\begin{array}{l}p-7 \\ n-1\end{array}\right)-2\left(\begin{array}{l}p-10 \\ n-1\end{array}\right)$.

Since $C_{n}(G, 2)=C_{n}(V, G, 2)+C_{n}(U, G, 2)$, then from (2.7) and (2.11), we get (2.2). \#

By the method used in proving Proposition 2.2, we get $C_{n}(G, 3)$.

Proposition 2.3: For $2 \leq \mathrm{n} \leq \mathrm{p}$ and $\alpha, \beta \geq 7$, we have

$$
\begin{array}{r}
\mathrm{C}_{\mathrm{n}}(\mathrm{G}, 3)=(\beta-4)\left(\begin{array}{l}
\mathrm{p}-5 \\
\mathrm{n}-1
\end{array}\right)+(\alpha-5)\left(\begin{array}{l}
\beta \\
\mathrm{n}-1
\end{array}\right)-(\alpha-8)\left(\begin{array}{l}
\beta-2 \\
\mathrm{n}-1
\end{array}\right)-\left(\begin{array}{l}
\beta-4 \\
\mathrm{n}-1
\end{array}\right)-2\left(\begin{array}{l}
\beta-6 \\
\mathrm{n}-1
\end{array}\right) \\
-(\beta-8)\left(\begin{array}{l}
\mathrm{p}-7 \\
\mathrm{n}-1
\end{array}\right)-2\left(\begin{array}{l}
\mathrm{p}-9 \\
\mathrm{n}-1
\end{array}\right)+2\left(\begin{array}{l}
\mathrm{p}-10 \\
\mathrm{n}-1
\end{array}\right)-2\left(\begin{array}{l}
\mathrm{p}-12 \\
\mathrm{n}-1
\end{array}\right)-2\left(\begin{array}{l}
\beta-7 \\
\mathrm{n}-1
\end{array}\right)
\end{array}
$$

Proof: From Fig. 2.1 and by using a procedure that is used in proving Proposition 2.2, we get:

$$
\begin{aligned}
& C_{n}\left(v_{1}, G, 3\right)=\left(\begin{array}{l}
\beta-2 \\
n-1
\end{array}\right)-\left(\begin{array}{l}
\beta-4 \\
n-1
\end{array}\right), \\
& C_{n}\left(v_{2}, G, 3\right)=C_{n}\left(v_{\alpha}, G, 3\right)=\left(\begin{array}{l}
\beta-4 \\
n-1
\end{array}\right)-\left(\begin{array}{l}
\beta-6 \\
n-1
\end{array}\right) \\
& C_{n}\left(v_{3}, G, 3\right)=C_{n}\left(v_{\alpha-1}, G, 3\right)=\left(\begin{array}{l}
\beta-2 \\
n-1
\end{array}\right)-\left(\begin{array}{l}
\beta-4 \\
n-1
\end{array}\right), \\
& C_{n}\left(v_{i}, G, 3\right)=\left(\begin{array}{c}
\beta \\
n-1
\end{array}\right)-\left(\begin{array}{l}
\beta-2 \\
n-1
\end{array}\right), i=4,5, \ldots, \alpha-2 .
\end{aligned}
$$

Thus,

$$
C_{n}(V, G, 3)=(\alpha-5)\left(\begin{array}{c}
\beta \\
n-1
\end{array}\right)-(\alpha-8)\left(\begin{array}{l}
\beta-2 \\
n-1
\end{array}\right)-\left(\begin{array}{l}
\beta-4 \\
n-1
\end{array}\right)-2\left(\begin{array}{l}
\beta-6 \\
n-1
\end{array}\right) \text {. }
$$

Moreover, 


$$
\begin{aligned}
& C_{n}\left(u_{1}, G, 3\right)=C_{n}\left(u_{\beta}, G, 3\right)=\left(\begin{array}{c}
p-10 \\
n-1
\end{array}\right)-\left(\begin{array}{c}
\beta-7 \\
n-1
\end{array}\right), \\
& C_{n}\left(u_{2}, G, 3\right)=C_{n}\left(u_{\beta-1}, G, 3\right)=\left(\begin{array}{c}
p-7 \\
n-1
\end{array}\right)-\left(\begin{array}{c}
p-12 \\
n-1
\end{array}\right), \\
& C_{n}\left(u_{3}, G, 3\right)=C_{n}\left(u_{\beta-2}, G, 3\right)=\left(\begin{array}{l}
p-5 \\
n-1
\end{array}\right)-\left(\begin{array}{l}
p-9 \\
n-1
\end{array}\right), \\
& C_{n}\left(u_{i}, G, 3\right)=\left(\begin{array}{c}
p-5 \\
n-1
\end{array}\right)-\left(\begin{array}{l}
p-7 \\
n-1
\end{array}\right), i=4,5, \ldots, \beta-3 .
\end{aligned}
$$

Thus,

$$
\begin{aligned}
\mathrm{C}_{\mathrm{n}}(\mathrm{U}, \mathrm{G}, 3)=(\beta-4)\left(\begin{array}{l}
\mathrm{p}-5 \\
\mathrm{n}-1
\end{array}\right)-(\beta-8)\left(\begin{array}{l}
\mathrm{p}-7 \\
\mathrm{n}-1
\end{array}\right)-2\left(\begin{array}{l}
\mathrm{p}-12 \\
\mathrm{n}-1
\end{array}\right)-2\left(\begin{array}{l}
\beta-7 \\
\mathrm{n}-1
\end{array}\right) \\
+2\left(\begin{array}{l}
\mathrm{p}-10 \\
\mathrm{n}-1
\end{array}\right)-2\left(\begin{array}{l}
\mathrm{p}-9 \\
\mathrm{n}-1
\end{array}\right) .
\end{aligned}
$$

Since $C_{n}(G, 3)=C_{n}(V, G, 3)+C_{n}(U, G, 3)$, then from (2.13) and (2.14), we obtain (2.12). \#

We shall obtain $C_{n}(V, G, k)$ and $C_{n}(U, G, k)$, for $4 \leq k \leq \operatorname{diam}_{n}(G)=\delta_{n}$.

We assume that $3 \leq n \leq p$, is the order of $G$, see Fig, 2.1.

It is clear that

$\mathrm{N}_{\mathrm{k}}^{=}\left(\mathrm{v}_{2}\right)=2$ and $\mathrm{N}_{\mathrm{k}}^{+}\left(\mathrm{v}_{2}\right)=\beta-2 \mathrm{k}$, for $4 \leq \mathrm{k} \leq \delta_{\mathrm{n}}-2$, and

$\mathrm{N}_{\mathrm{k}}^{=}\left(\mathrm{v}_{2}\right)=\mathrm{N}_{\mathrm{k}}^{+}\left(\mathrm{v}_{2}\right)=0$, for $\mathrm{k}=\delta_{\mathrm{n}}-1$ and $\delta_{\mathrm{n}} \geq 6$.

Thus,

$$
\mathrm{C}_{\mathrm{n}}\left(\mathrm{v}_{2}, \mathrm{G}, \mathrm{k}\right)=\left(\begin{array}{c}
\beta-2 \mathrm{k}+2 \\
\mathrm{n}-1
\end{array}\right)-\left(\begin{array}{c}
\beta-2 \mathrm{k} \\
\mathrm{n}-1
\end{array}\right)=\mathrm{C}_{\mathrm{n}}\left(\mathrm{v}_{\alpha}, \mathrm{G}, \mathrm{k}\right) \text {, for } 4 \leq \mathrm{k} \leq \delta_{\mathrm{n}} .
$$

Similarly, for $4 \leq \mathrm{k} \leq \delta_{\mathrm{n}}-1,\left(\delta_{\mathrm{n}} \geq 5\right)$

$\mathrm{N}_{\mathrm{k}}^{=}\left(\mathrm{v}_{1}\right)=2$ and $\mathrm{N}_{\mathrm{k}}^{+}\left(\mathrm{v}_{1}\right)=\beta-2 \mathrm{k}+2$,

$\mathrm{N}_{\delta_{\mathrm{n}}}^{=}\left(\mathrm{v}_{1}\right)=\mathrm{N}_{\delta_{\mathrm{n}}}^{+}\left(\mathrm{v}_{1}\right)=0$, therefore, for $4 \leq \mathrm{k} \leq \delta_{\mathrm{n}}$, we have

$\mathrm{C}_{\mathrm{n}}\left(\mathrm{v}_{1}, \mathrm{G}, \mathrm{k}\right)=\mathrm{C}_{\mathrm{n}}\left(\mathrm{v}_{3}, \mathrm{G}, \mathrm{k}\right)=\mathrm{C}_{\mathrm{n}}\left(\mathrm{v}_{\alpha-1}, \mathrm{G}, \mathrm{k}\right)=\left(\begin{array}{c}\beta-2 \mathrm{k}+4 \\ \mathrm{n}-1\end{array}\right)-\left(\begin{array}{c}\beta-2 \mathrm{k}+2 \\ \mathrm{n}-1\end{array}\right)$.

Finally, for $4 \leq \mathrm{k} \leq \delta_{\mathrm{n}}$ and $\mathrm{i}=4,5, \ldots, \alpha-2$

$\mathrm{N}_{\mathrm{k}}^{=}\left(\mathrm{v}_{\mathrm{i}}\right)=2$ and $\mathrm{N}_{\mathrm{k}}^{+}\left(\mathrm{v}_{\mathrm{i}}\right)=\beta-2 \mathrm{k}+4$, thus for $4 \leq \mathrm{k} \leq \delta_{\mathrm{n}}$, we have

$C_{n}\left(v_{i}, G, k\right)=\left(\begin{array}{c}\beta-2 k+6 \\ n-1\end{array}\right)-\left(\begin{array}{c}\beta-2 k+4 \\ n-1\end{array}\right)$, for $\mathrm{i}=4,5, \ldots, \alpha-2$.

Hence, from (2.15), (2.16) and (2.17), we have

Proposition 2.4: For $4 \leq \mathrm{k} \leq \delta_{\mathrm{n}}$ and $3 \leq \mathrm{n} \leq \mathrm{p}$,

$C_{n}(V, G, k)=(\alpha-5)\left(\begin{array}{c}\beta-2 k+6 \\ n-1\end{array}\right)-(\alpha-8)\left(\begin{array}{c}\beta-2 k+4 \\ n-1\end{array}\right)-\left(\begin{array}{c}\beta-2 k+2 \\ n-1\end{array}\right)-2\left(\begin{array}{c}\beta-2 k \\ n-1\end{array}\right)$

Remark (1): It is clear from Proposition 2.1 that for all values of $n$, 
$\delta_{n}=\operatorname{diam}_{n}(G) \leq\left\lfloor\frac{\beta-1}{2}\right\rfloor+3=\left[\begin{array}{ll}\frac{\beta}{2}+2, & \text { for even } \beta, \\ \frac{\beta-1}{2}+3, & \text { for odd } \beta .\end{array}\right.$

To find $\mathrm{C}_{\mathrm{n}}(\mathrm{U}, \mathrm{G}, \mathrm{k}), 4 \leq \mathrm{k} \leq \delta_{\mathrm{n}}$, we shall consider the main two cases for $\beta$, namely even or odd .

(a). Let $\beta$ be even and denoted $t=\beta / 2$ :

From Fig.2.1, we get for $\mathrm{j}=1,2, \ldots, \mathrm{k}-3$, and $\mathrm{k}=4,5, \ldots, \mathrm{t}-1$

$\mathrm{N}_{\mathrm{k}}^{=}\left(\mathrm{u}_{\mathrm{j}}\right)=2$ and $\mathrm{N}_{\mathrm{k}}^{+}\left(\mathrm{u}_{\mathrm{j}}\right)=\beta-2 \mathrm{k}-1$, therefore, for $\mathrm{k}=4,5, \ldots, \mathrm{t}-1$

$C_{n}\left(u_{j}, G, k\right)=C_{n}\left(u_{\beta-j+1}, G, k\right)=\left(\begin{array}{c}\beta-2 k+1 \\ n-1\end{array}\right)-\left(\begin{array}{c}\beta-2 k-1 \\ n-1\end{array}\right), j=1,2, \ldots, k-3$.

Also, for $\mathrm{k}=4,5, \ldots, \mathrm{t}-1$,

$\mathrm{N}_{\mathrm{k}}^{=}\left(\mathrm{u}_{\mathrm{k}-2}\right)=\alpha-3$ and $\mathrm{N}_{\mathrm{k}}^{+}\left(\mathrm{u}_{\mathrm{k}-2}\right)=\beta-2 \mathrm{k}-1$, therefore

$\mathrm{C}_{\mathrm{n}}\left(\mathrm{u}_{\mathrm{k}-2}, \mathrm{G}, \mathrm{k}\right)=\mathrm{C}_{\mathrm{n}}\left(\mathrm{u}_{\beta-\mathrm{k}+3}, \mathrm{G}, \mathrm{k}\right)=\left(\begin{array}{c}\mathrm{p}-2 \mathrm{k}-4 \\ \mathrm{n}-1\end{array}\right)-\left(\begin{array}{c}\beta-2 \mathrm{k}-1 \\ \mathrm{n}-1\end{array}\right)$, for $4 \leq \mathrm{k} \leq \mathrm{t}-1$.

Similarly, for the same values of $k$, namely $4 \leq \mathrm{k} \leq \mathrm{t}-1$,

$\mathrm{N}_{\mathrm{k}}^{=}\left(\mathrm{u}_{\mathrm{k}-1}\right)=5$ and $\mathrm{N}_{\mathrm{k}}^{+}\left(\mathrm{u}_{\mathrm{k}-1}\right)=\mathrm{p}-2 \mathrm{k}-6$, therefore

$\mathrm{C}_{\mathrm{n}}\left(\mathrm{u}_{\mathrm{k}-1}, \mathrm{G}, \mathrm{k}\right)=\mathrm{C}_{\mathrm{n}}\left(\mathrm{u}_{\beta-\mathrm{k}+2}, \mathrm{G}, \mathrm{k}\right)=\left(\begin{array}{c}\mathrm{p}-2 \mathrm{k}-1 \\ \mathrm{n}-1\end{array}\right)-\left(\begin{array}{c}\mathrm{p}-2 \mathrm{k}-6 \\ \mathrm{n}-1\end{array}\right)$, for $4 \leq \mathrm{k} \leq \mathrm{t}-1$.

Also, $\mathrm{N}_{\mathrm{k}}^{=}\left(\mathrm{u}_{\mathrm{k}}\right)=4$ and $\mathrm{N}_{\mathrm{k}}^{+}\left(\mathrm{u}_{\mathrm{k}}\right)=\mathrm{p}-2 \mathrm{k}-3$, therefore

$\mathrm{C}_{\mathrm{n}}\left(\mathrm{u}_{\mathrm{k}}, \mathrm{G}, \mathrm{k}\right)=\mathrm{C}_{\mathrm{n}}\left(\mathrm{u}_{\beta-\mathrm{k}+1}, \mathrm{G}, \mathrm{k}\right)=\left(\begin{array}{c}\mathrm{p}-2 \mathrm{k}+1 \\ \mathrm{n}-1\end{array}\right)-\left(\begin{array}{c}\mathrm{p}-2 \mathrm{k}-3 \\ \mathrm{n}-1\end{array}\right)$, for $4 \leq \mathrm{k} \leq \mathrm{t}-1$.

Moreover, for $\mathrm{j}=\mathrm{k}+1, \mathrm{k}+2, \ldots, \beta / 2(=\mathrm{t})$, we have

$\mathrm{N}_{\mathrm{k}}^{=}\left(\mathrm{u}_{\mathrm{j}}\right)=2$ and $\mathrm{N}_{\mathrm{k}}^{+}\left(\mathrm{u}_{\mathrm{j}}\right)=\mathrm{p}-2 \mathrm{k}-1$, for $4 \leq \mathrm{k} \leq \mathrm{t}-1$.

Therefore, for $4 \leq \mathrm{k} \leq \mathrm{t}-1$, we get

$$
\begin{array}{r}
C_{n}\left(u_{j}, G, k\right)=C_{n}\left(u_{\beta-j+1}, G, k\right)=\left(\begin{array}{c}
p-2 k+1 \\
n-1
\end{array}\right)-\left(\begin{array}{c}
p-2 k-1 \\
n-1
\end{array}\right), \\
\text { for } j=k+1, k+2, \ldots, \beta / 2(=t) .
\end{array}
$$

From (2.19) - (2.23), we obtain the following statement .

Proposition 2.5: For $4 \leq \mathrm{k} \leq \mathrm{t}-1$ and $3 \leq \mathrm{n} \leq \mathrm{p}$, we have

$$
\begin{array}{r}
\mathrm{C}_{\mathrm{n}}(\mathrm{U}, \mathrm{G}, \mathrm{k})=2(\mathrm{k}-3)\left(\begin{array}{c}
\beta-2 \mathrm{k}+1 \\
\mathrm{n}-1
\end{array}\right)+2\left(\begin{array}{c}
\beta-2 \mathrm{k}-4 \\
\mathrm{n}-1
\end{array}\right)+(\beta-2 \mathrm{k}+2)\left(\begin{array}{c}
\mathrm{p}-2 \mathrm{k}+1 \\
\mathrm{n}-1
\end{array}\right) \\
-2(\mathrm{k}-2)\left(\begin{array}{c}
\beta-2 \mathrm{k}-1 \\
\mathrm{n}-1
\end{array}\right)-(\beta-2 \mathrm{k}-2)\left(\begin{array}{c}
\mathrm{p}-2 \mathrm{k}-1 \\
\mathrm{n}-1
\end{array}\right)-2\left(\begin{array}{c}
\mathrm{p}-2 \mathrm{k}-6 \\
\mathrm{n}-1
\end{array}\right) \\
-2\left(\begin{array}{c}
\mathrm{p}-2 \mathrm{k}-3 \\
\mathrm{n}-1
\end{array}\right) .
\end{array}
$$

For the other values of $\mathrm{k}$, we have the following result : 
Proposition 2.6: For $\mathrm{k}=\mathrm{t}, \mathrm{t}+1$ and $\mathrm{t}+2,3 \leq \mathrm{n} \leq \mathrm{p}$, we have:

(I). $C_{n}(U, G, t)=2\left[\left(\begin{array}{l}\alpha+1 \\ n-1\end{array}\right)+\left(\begin{array}{l}\alpha-1 \\ n-1\end{array}\right)+\left(\begin{array}{l}\alpha-4 \\ n-1\end{array}\right)-\left(\begin{array}{l}\alpha-2 \\ n-1\end{array}\right)-\left(\begin{array}{l}\alpha-5 \\ n-1\end{array}\right)\right]$.

(II). $C_{n}(U, G, t+1)=2\left(\begin{array}{l}\alpha-2 \\ n-1\end{array}\right)$.

(III). $\mathrm{C}_{\mathrm{n}}(\mathrm{U}, \mathrm{G}, \mathrm{t}+2)=2\left(\begin{array}{c}\alpha-5 \\ \mathrm{n}-1\end{array}\right)$.

Proof : (I) For $\mathrm{k}=\mathrm{t}$, we have :

$\mathrm{N}_{\mathrm{t}}^{=}\left(\mathrm{u}_{\mathrm{i}}\right)=1$ and $\mathrm{N}_{\mathrm{t}}^{+}\left(\mathrm{u}_{\mathrm{i}}\right)=0$, for $\mathrm{i}=1,2, \ldots, \mathrm{t}-3$, thus

$C_{n}\left(u_{i}, G, t\right)=C_{n}\left(u_{\beta-i+1}, G, t\right)=0$, for $i=1,2, \ldots, t-3$.

$\mathrm{N}_{\mathrm{t}}^{=}\left(\mathrm{u}_{\mathrm{t}-2}\right)=\alpha-4$ and $\mathrm{N}_{\mathrm{t}}^{+}\left(\mathrm{u}_{\mathrm{t}-2}\right)=0$, thus

$\mathrm{C}_{\mathrm{n}}\left(\mathrm{u}_{\mathrm{t}-2}, \mathrm{G}, \mathrm{t}\right)=\mathrm{C}_{\mathrm{n}}\left(\mathrm{u}_{\beta-\mathrm{t}+3}, \mathrm{G}, \mathrm{t}\right)=\left(\begin{array}{c}\alpha-4 \\ \mathrm{n}-1\end{array}\right)$.

$\mathrm{N}_{\mathrm{t}}^{=}\left(\mathrm{u}_{\mathrm{t}-1}\right)=4$ and $\mathrm{N}_{\mathrm{t}}^{+}\left(\mathrm{u}_{\mathrm{t}-1}\right)=\alpha-5$, thus

$\mathrm{C}_{\mathrm{n}}\left(\mathrm{u}_{\mathrm{t}-1}, \mathrm{G}, \mathrm{t}\right)=\mathrm{C}_{\mathrm{n}}\left(\mathrm{u}_{\beta-\mathrm{t}+2}, \mathrm{G}, \mathrm{t}\right)=\left(\begin{array}{c}\alpha-1 \\ \mathrm{n}-1\end{array}\right)-\left(\begin{array}{c}\alpha-5 \\ \mathrm{n}-1\end{array}\right)$.

$\mathrm{N}_{\mathrm{t}}^{=}\left(\mathrm{u}_{\mathrm{t}}\right)=3$ and $\mathrm{N}_{\mathrm{t}}^{+}\left(\mathrm{u}_{\mathrm{t}}\right)=\alpha-2$, thus

$C_{n}\left(u_{t}, G, t\right)=C_{n}\left(u_{\beta-t+1}, G, t\right)=\left(\begin{array}{c}\alpha+1 \\ n-1\end{array}\right)-\left(\begin{array}{c}\alpha-2 \\ n-1\end{array}\right)$.

Hence, from (2.25.a) - (2.25.d), we obtain (2.25) .

(II) For $\mathrm{k}=\mathrm{t}+1$, we have :

$\mathrm{N}_{\mathrm{t}+1}^{=}\left(\mathrm{u}_{\mathrm{i}}\right)=\mathrm{N}_{\mathrm{t}+1}^{+}\left(\mathrm{u}_{\mathrm{i}}\right)=0$, for $\mathrm{i}=1,2, \ldots, \mathrm{t}-2$.

$\mathrm{C}_{\mathrm{n}}\left(\mathrm{u}_{\mathrm{i}}, \mathrm{G}, \mathrm{t}+1\right)=\mathrm{C}_{\mathrm{n}}\left(\mathrm{u}_{\beta-\mathrm{i}+1}, \mathrm{G}, \mathrm{t}+1\right)=0$, for $\mathrm{i}=1,2, \ldots, \mathrm{t}-2$.

$\mathrm{N}_{\mathrm{t}+1}^{=}\left(\mathrm{u}_{\mathrm{t}-1}\right)=\alpha-5$ and $\mathrm{N}_{\mathrm{t}+1}^{+}\left(\mathrm{u}_{\mathrm{t}-1}\right)=0$, thus

$\mathrm{C}_{\mathrm{n}}\left(\mathrm{u}_{\mathrm{t}-1}, \mathrm{G}, \mathrm{t}+1\right)=\mathrm{C}_{\mathrm{n}}\left(\mathrm{u}_{\beta-\mathrm{t}+2}, \mathrm{G}, \mathrm{t}+1\right)=\left(\begin{array}{c}\alpha-5 \\ \mathrm{n}-1\end{array}\right)$.

$\mathrm{N}_{\mathrm{t}+1}^{=}\left(\mathrm{u}_{\mathrm{t}}\right)=3$ and $\mathrm{N}_{\mathrm{t}+1}^{+}\left(\mathrm{u}_{\mathrm{t}}\right)=\alpha-5$, thus

$\mathrm{C}_{\mathrm{n}}\left(\mathrm{u}_{\mathrm{t}}, \mathrm{G}, \mathrm{t}+1\right)=\mathrm{C}_{\mathrm{n}}\left(\mathrm{u}_{\beta-\mathrm{t}+1}, \mathrm{G}, \mathrm{t}+1\right)=\left(\begin{array}{l}\alpha-2 \\ \mathrm{n}-1\end{array}\right)-\left(\begin{array}{l}\alpha-5 \\ \mathrm{n}-1\end{array}\right)$.

Hence, from (2.26.a), (2.26.b) and (2.26.c) we obtain (2.26) .

(III) For $\mathrm{k}=\mathrm{t}+2$, we have :

$\mathrm{N}_{\mathrm{t}+2}^{=}\left(\mathrm{u}_{\mathrm{i}}\right)=\mathrm{N}_{\mathrm{t}+2}^{+}\left(\mathrm{u}_{\mathrm{i}}\right)=0$, for $\mathrm{i}=1,2, \ldots, \mathrm{t}-1$.

$\mathrm{N}_{\mathrm{t}+2}^{=}\left(\mathrm{u}_{\mathrm{t}}\right)=\alpha-5$ and $\mathrm{N}_{\mathrm{t}+2}^{+}\left(\mathrm{u}_{\mathrm{t}}\right)=0$, thus

$\mathrm{C}_{\mathrm{n}}\left(\mathrm{u}_{\mathrm{t}}, \mathrm{G}, \mathrm{t}+2\right)=\left(\begin{array}{l}\alpha-5 \\ \mathrm{n}-1\end{array}\right)$.

Hence, from (2.27.a) and (2.27.b), we obtain (2.27). This completes the proof .\# 


\section{(b). Let $\beta$ be odd :}

Remark (2): One may check that Proposition 2.5 holds for odd $\beta$ and for $4 \leq \mathrm{k} \leq \frac{\beta-1}{2}$, that is $\mathrm{C}_{\mathrm{n}}(\mathrm{U}, \mathrm{G}, \mathrm{k})$ is given in (2.24) for odd $\beta$ and $4 \leq \mathrm{k} \leq \frac{\beta-1}{2}$.

Let $\mathrm{t}^{\prime}=\frac{\beta-1}{2}$, where $\beta$ is odd,$\beta \geq 9$.

Proposition 2.7: For odd $\beta$ and $\mathrm{k}=\mathrm{t}^{\prime}+1, \mathrm{t}^{\prime}+2$ and $\mathrm{t}^{\prime}+3,3 \leq \mathrm{n} \leq \mathrm{p}$, we have:

$\left(I^{\prime}\right) \cdot C_{n}\left(U, G, t^{\prime}+1\right)=\left(\begin{array}{c}\alpha \\ n-1\end{array}\right)+\left(\begin{array}{c}\alpha-2 \\ n-1\end{array}\right)$.

$\left(\mathrm{II}^{\prime}\right) \cdot \mathrm{C}_{\mathrm{n}}\left(\mathrm{U}, \mathrm{G}, \mathrm{t}^{\prime}+2\right)=\left(\begin{array}{l}\alpha-2 \\ \mathrm{n}-1\end{array}\right)+\left(\begin{array}{l}\alpha-5 \\ \mathrm{n}-1\end{array}\right)$.

$\left(\mathrm{III}^{\prime}\right) \cdot \mathrm{C}_{\mathrm{n}}\left(\mathrm{U}, \mathrm{G}, \mathrm{t}^{\prime}+3\right)=\left(\begin{array}{c}\alpha-5 \\ \mathrm{n}-1\end{array}\right)$.

Proof: For each vertex $u_{j}$, there is no vertex of $U$ which is of distance $t^{\prime}+1, t^{\prime}+2$ or $t^{\prime}+3$ from $u_{j}$. Therefore, the only vertices of distance $t^{\prime}+1, t^{\prime}+2$ or $t^{\prime}+3$ from each $u_{j}$ are in $W_{\alpha}$. Moreover, the vertices of $U$ that are of distance $t^{\prime}+1, t^{\prime}+2$ or $\mathrm{t}^{\prime}+3$ from vertices of $\mathrm{W}_{\alpha}$ are $\mathrm{u}_{\mathrm{t}^{\prime}-1}, \mathrm{u}_{\mathrm{t}^{\prime}}$ and $\mathrm{u}_{\mathrm{t}^{\prime}+1}$, (and by symmetry $\mathrm{u}_{\mathrm{t}^{\prime}+2}$ and $\mathrm{u}_{\mathrm{t}^{\prime}+3}$ ).

$\left(I^{\prime}\right)$. For $k=t^{\prime}+1$, we have

$\mathrm{N}_{\mathrm{t}^{\prime}+1}^{=}\left(\mathrm{u}_{\mathrm{t}^{\prime}-1}\right)=\alpha-5$ and $\mathrm{N}_{\mathrm{t}^{\prime}+1}^{+}\left(\mathrm{u}_{\mathrm{t}^{\prime}-1}\right)=0$, thus

$\mathrm{C}_{\mathrm{n}}\left(\mathrm{u}_{\mathrm{t}^{\prime}-1}, \mathrm{G}, \mathrm{t}^{\prime}+1\right)=\mathrm{C}_{\mathrm{n}}\left(\mathrm{u}_{\mathrm{t}^{\prime}+3}, \mathrm{G}, \mathrm{t}^{\prime}+1\right)=\left(\begin{array}{c}\alpha-5 \\ \mathrm{n}-1\end{array}\right)$.

$\mathrm{N}_{\mathrm{t}^{\prime}+1}^{=}\left(\mathrm{u}_{\mathrm{t}^{\prime}}\right)=3$ and $\mathrm{N}_{\mathrm{t}^{\prime}+1}^{+}\left(\mathrm{u}_{\mathrm{t}^{\prime}}\right)=\alpha-5$, thus

$\mathrm{C}_{\mathrm{n}}\left(\mathrm{u}_{\mathrm{t}^{\prime}}, \mathrm{G}, \mathrm{t}^{\prime}+1\right)=\mathrm{C}_{\mathrm{n}}\left(\mathrm{u}_{\mathrm{t}^{\prime}+2}, \mathrm{G}, \mathrm{t}^{\prime}+1\right)=\left(\begin{array}{c}\alpha-2 \\ \mathrm{n}-1\end{array}\right)-\left(\begin{array}{c}\alpha-5 \\ \mathrm{n}-1\end{array}\right)$.

$\mathrm{N}_{\mathrm{t}^{\prime}+1}^{=}\left(\mathrm{u}_{\mathrm{t}^{\prime}+1}\right)=2$ and $\mathrm{N}_{\mathrm{t}^{\prime}+1}^{+}\left(\mathrm{u}_{\mathrm{t}^{\prime}+1}\right)=\alpha-2$, thus

$\mathrm{C}_{\mathrm{n}}\left(\mathrm{u}_{\mathrm{t}^{\prime}+1}, \mathrm{G}, \mathrm{t}^{\prime}+1\right)=\left(\begin{array}{c}\alpha \\ \mathrm{n}-1\end{array}\right)-\left(\begin{array}{c}\alpha-2 \\ \mathrm{n}-1\end{array}\right)$.

Hence, from (2.28.a), (2.28.b) and (2.28.c) we obtain (2.28) .

( II'). For $\mathrm{k}=\mathrm{t}^{\prime}+2$, we have

$\mathrm{N}_{\mathrm{t}^{\prime}+2}^{=}\left(\mathrm{u}_{\mathrm{t}^{\prime}-1}\right)=\mathrm{N}_{\mathrm{t}^{\prime}+2}^{+}\left(\mathrm{u}_{\mathrm{t}^{\prime}-1}\right)=0$, thus

$\mathrm{C}_{\mathrm{n}}\left(\mathrm{u}_{\mathrm{t}^{\prime}-1}, \mathrm{G}, \mathrm{t}^{\prime}+2\right)=\mathrm{C}_{\mathrm{n}}\left(\mathrm{u}_{\mathrm{t}^{\prime}+3}, \mathrm{G}, \mathrm{t}^{\prime}+2\right)=0$.

$\mathrm{N}_{\mathrm{t}^{\prime}+2}^{=}\left(\mathrm{u}_{\mathrm{t}^{\prime}}\right)=\alpha-5$ and $\mathrm{N}_{\mathrm{t}^{\prime}+2}^{+}\left(\mathrm{u}_{\mathrm{t}^{\prime}}\right)=0$, thus

$\mathrm{C}_{\mathrm{n}}\left(\mathrm{u}_{\mathrm{t}^{\prime}}, \mathrm{G}, \mathrm{t}^{\prime}+2\right)=\mathrm{C}_{\mathrm{n}}\left(\mathrm{u}_{\mathrm{t}^{\prime}+2}, \mathrm{G}, \mathrm{t}^{\prime}+2\right)=\left(\begin{array}{c}\alpha-5 \\ \mathrm{n}-1\end{array}\right)$.

$\mathrm{N}_{\mathrm{t}^{\prime}+2}^{=}\left(\mathrm{u}_{\mathrm{t}^{\prime}+1}\right)=3$ and $\mathrm{N}_{\mathrm{t}^{\prime}+2}^{+}\left(\mathrm{u}_{\mathrm{t}^{\prime}+1}\right)=\alpha-5$, thus 
$C_{n}\left(u_{t^{\prime}+1}, G, t^{\prime}+2\right)=\left(\begin{array}{l}\alpha-2 \\ n-1\end{array}\right)-\left(\begin{array}{l}\alpha-5 \\ n-1\end{array}\right)$.

Hence, from (2.29.a), (2.29.b) and (2.29.c) we obtain (2.29).

( III' ). For $\mathrm{k}=\mathrm{t}^{\prime}+3$, we have

$\mathrm{N}_{\mathrm{t}^{\prime}+3}^{=}\left(\mathrm{u}_{\mathrm{t}^{\prime}-1}\right)=\mathrm{N}_{\mathrm{t}^{\prime}+3}^{+}\left(\mathrm{u}_{\mathrm{t}^{\prime}-1}\right)=0$, thus

$\mathrm{C}_{\mathrm{n}}\left(\mathrm{u}_{\mathrm{t}^{\prime}-1}, \mathrm{G}, \mathrm{t}^{\prime}+3\right)=\mathrm{C}_{\mathrm{n}}\left(\mathrm{u}_{\mathrm{t}^{\prime}+3}, \mathrm{G}, \mathrm{t}^{\prime}+3\right)=0$.

$\mathrm{N}_{\mathrm{t}^{\prime}+3}^{=}\left(\mathrm{u}_{\mathrm{t}^{\prime}}\right)=\mathrm{N}_{\mathrm{t}^{\prime}+3}^{+}\left(\mathrm{u}_{\mathrm{t}^{\prime}}\right)=0$, thus

$\mathrm{C}_{\mathrm{n}}\left(\mathrm{u}_{\mathrm{t}^{\prime}}, \mathrm{G}, \mathrm{t}^{\prime}+3\right)=\mathrm{C}_{\mathrm{n}}\left(\mathrm{u}_{\mathrm{t}^{\prime}+2}, \mathrm{G}, \mathrm{t}^{\prime}+3\right)=0$.

$\mathrm{N}_{\mathrm{t}^{\prime}+3}^{=}\left(\mathrm{u}_{\mathrm{t}^{\prime}+1}\right)=\alpha-5$ and $\mathrm{N}_{\mathrm{t}^{\prime}+3}^{+}\left(\mathrm{u}_{\mathrm{t}^{\prime}+1}\right)=0$, thus

$\mathrm{C}_{\mathrm{n}}\left(\mathrm{u}_{\mathrm{t}^{\prime}+1}, \mathrm{G}, \mathrm{t}^{\prime}+3\right)=\left(\begin{array}{c}\alpha-5 \\ \mathrm{n}-1\end{array}\right)$.

Hence, from (2.30.a), (2.30.b) and (2.30.c) we obtain (2.30).

This completes the proof .\#

From (2.18) and (2.24), we obtain $\mathrm{C}_{\mathrm{n}}(\mathrm{G}, \mathrm{k})$ for $4 \leq \mathrm{k} \leq \mathrm{t}-1$ for even $\beta$ as given next :

$\mathrm{C}_{\mathrm{n}}(\mathrm{G}, \mathrm{k})=\mathrm{C}_{\mathrm{n}}(\mathrm{U}, \mathrm{G}, \mathrm{k})+\mathrm{C}_{\mathrm{n}}(\mathrm{V}, \mathrm{G}, \mathrm{k}), 4 \leq \mathrm{k} \leq \mathrm{t}-1$, even $\beta$,

$$
\begin{array}{r}
=(\alpha-5)\left(\begin{array}{c}
\beta-2 \mathrm{k}+6 \\
\mathrm{n}-1
\end{array}\right)+2(\mathrm{k}-3)\left(\begin{array}{c}
\beta-2 \mathrm{k}+1 \\
\mathrm{n}-1
\end{array}\right)+2\left(\begin{array}{c}
\mathrm{p}-2 \mathrm{k}-4 \\
\mathrm{n}-1
\end{array}\right)+(\beta-2 \mathrm{k}+2)\left(\begin{array}{c}
\mathrm{p}-2 \mathrm{k}+1 \\
\mathrm{n}-1
\end{array}\right) \\
-(\alpha-8)\left(\begin{array}{c}
\beta-2 \mathrm{k}+4 \\
\mathrm{n}-1
\end{array}\right)-\left(\begin{array}{c}
\beta-2 \mathrm{k}+2 \\
\mathrm{n}-1
\end{array}\right)-2\left(\begin{array}{c}
\beta-2 \mathrm{k} \\
\mathrm{n}-1
\end{array}\right)-2(\mathrm{k}-2)\left(\begin{array}{c}
\beta-2 \mathrm{k}-1 \\
\mathrm{n}-1
\end{array}\right) \\
-(\beta-2 \mathrm{k}-2)\left(\begin{array}{c}
\mathrm{p}-2 \mathrm{k}-1 \\
\mathrm{n}-1
\end{array}\right)-2\left(\begin{array}{c}
\mathrm{p}-2 \mathrm{k}-6 \\
\mathrm{n}-1
\end{array}\right)-2\left(\begin{array}{c}
\mathrm{p}-2 \mathrm{k}-3 \\
\mathrm{n}-1
\end{array}\right) .
\end{array}
$$

For other values of $\mathrm{k}$, we have from (2.18) and (2.25)-(2.28):

$$
\begin{aligned}
& \mathrm{C}_{\mathrm{n}}(\mathrm{G}, \mathrm{t})=2\left[\left(\begin{array}{l}
\alpha+1 \\
\mathrm{n}-1
\end{array}\right)+\left(\begin{array}{l}
\alpha-1 \\
\mathrm{n}-1
\end{array}\right)+\left(\begin{array}{c}
\alpha-4 \\
\mathrm{n}-1
\end{array}\right)-\left(\begin{array}{c}
\alpha-2 \\
\mathrm{n}-1
\end{array}\right)-\left(\begin{array}{c}
\alpha-5 \\
\mathrm{n}-1
\end{array}\right)\right]+(\alpha-5)\left(\begin{array}{c}
6 \\
\mathrm{n}-1
\end{array}\right) \\
& -(\alpha-8)\left(\begin{array}{c}
4 \\
\mathrm{n}-1
\end{array}\right)-\left(\begin{array}{c}
2 \\
\mathrm{n}-1
\end{array}\right) . \\
& \mathrm{C}_{\mathrm{n}}(\mathrm{G}, \mathrm{t}+1)=2\left(\begin{array}{l}
\alpha-2 \\
\mathrm{n}-1
\end{array}\right)+(\alpha-5)\left(\begin{array}{c}
4 \\
\mathrm{n}-1
\end{array}\right)-(\alpha-8)\left(\begin{array}{c}
2 \\
\mathrm{n}-1
\end{array}\right) . \\
& \mathrm{C}_{\mathrm{n}}(\mathrm{G}, \mathrm{t}+2)=2\left(\begin{array}{l}
\alpha-5 \\
\mathrm{n}-1
\end{array}\right)+(\alpha-5)\left(\begin{array}{c}
2 \\
\mathrm{n}-1
\end{array}\right) .
\end{aligned}
$$

The formula (2.31) holds also for odd $\beta$ and $4 \leq \mathrm{k} \leq \mathrm{t}^{\prime}=\frac{\beta-1}{2}$. This is required to find $C_{n}(G, k)$ for other values of $k$, namely $t^{\prime}+1, t^{\prime}+2$ and $t^{\prime}+3$.

Hence, for odd $\beta$ : 


$$
\begin{aligned}
& \mathrm{C}_{\mathrm{n}}\left(\mathrm{G}, \mathrm{t}^{\prime}+1\right)=(\alpha-5)\left(\begin{array}{c}
6 \\
\mathrm{n}-1
\end{array}\right)-(\alpha-8)\left(\begin{array}{c}
4 \\
\mathrm{n}-1
\end{array}\right)-2\left(\begin{array}{c}
2 \\
\mathrm{n}-1
\end{array}\right)+\left(\begin{array}{c}
\alpha \\
\mathrm{n}-1
\end{array}\right)+\left(\begin{array}{l}
\alpha-2 \\
\mathrm{n}-1
\end{array}\right) \\
& \mathrm{C}_{\mathrm{n}}\left(\mathrm{G}, \mathrm{t}^{\prime}+2\right)=(\alpha-5)\left(\begin{array}{c}
3 \\
\mathrm{n}-1
\end{array}\right)+\left(\begin{array}{l}
\alpha-2 \\
\mathrm{n}-1
\end{array}\right)+\left(\begin{array}{l}
\alpha-5 \\
\mathrm{n}-1
\end{array}\right) . \\
& \mathrm{C}_{\mathrm{n}}\left(\mathrm{G}, \mathrm{t}^{\prime}+3\right)=\left(\begin{array}{l}
\alpha-5 \\
\mathrm{n}-1
\end{array}\right) .
\end{aligned}
$$

Now, we can state the main theorem :

Theorem 2.8: For $3 \leq \mathrm{n} \leq \mathrm{p}(=\alpha+\beta), \alpha \geq 8, \beta \geq 10$ we have:

$$
\mathrm{H}_{\mathrm{n}}(\mathrm{G} ; \mathrm{x})=\sum_{\mathrm{k}=1}^{\delta_{\mathrm{n}}} \mathrm{C}_{\mathrm{n}}(\mathrm{G}, \mathrm{k}) \mathrm{x}^{\mathrm{k}} \text {, and } \mathrm{W}_{\mathrm{n}}(\mathrm{G})=\sum_{\mathrm{k}=1}^{\delta_{\mathrm{n}}} \mathrm{kC}_{\mathrm{n}}(\mathrm{G}, \mathrm{k})
$$

where $\delta_{\mathrm{n}}$ is the $\mathrm{n}$-diameter determined by Proposition 2.1 , and $\mathrm{C}_{\mathrm{n}}(\mathrm{G}, \mathrm{k})$ is given in (2.31) for $4 \leq \mathrm{k} \leq \frac{\beta}{2}-1$, for even $\beta ; \quad\left(4 \leq \mathrm{k} \leq \frac{\beta-1}{2}\right.$, for odd $\left.\beta\right)$, and for $\mathrm{k}=\frac{\beta}{2}, \frac{\beta}{2}+1$, and $\frac{\beta}{2}+2, \mathrm{C}_{\mathrm{n}}(\mathrm{G}, \mathrm{k})$ is given in $(2.32),(2.33)$ and (2.34), respectively, for even $\beta$; but for $\mathrm{k}=\frac{\beta+1}{2}, \frac{\beta+3}{2}$, and $\frac{\beta+5}{2}, \mathrm{C}_{\mathrm{n}}(\mathrm{G}, \mathrm{k})$ is given by $(2.35),(2.36)$ and (2.37) for odd $\beta$. For $\mathrm{k}=1,2$ and $3, \mathrm{C}_{\mathrm{n}}(\mathrm{G}, \mathrm{k})$ is given by (2.1), (2.2) and (2.3), respectively.\#

Remark (3): For $4 \leq \alpha \leq 7$ and $4 \leq \beta \leq 9$, one can easily find $H_{n}(G ; x)$ and $W_{n}(G)$ by direct calculation of $\mathrm{C}_{\mathrm{n}}(\mathrm{G}, \mathrm{k}), 1 \leq \mathrm{k} \leq 7$.

\section{REFERENCES}

[1] H.O. Abdulla (2007), Hosoya Polynomials of Steiner Distance of Some Graphs, Ph.D. Thesis, University of Salahaddin-Erbil / Iraq.

[2] H.G. Ahmed (2007), On Wiener Polynomials of n-Distance in Graphs, M.Sc. Thesis, University of Dohuk / Iraq.

[3] H.G. Ahmed; The n-Hosoya Polynomial of $\mathrm{W}_{\alpha} \otimes \mathrm{W}_{\beta}$; submitted for publication in J. of Dohuk University.

[4] A.M. Ali; (2005). Wiener Polynomials of Generalized Distance in Graphs, M.Sc. Thesis, Mosul University, Mosul. 
[5] A.M. Ali; The n-Hosoya Polynomials of the Composite of Some Special Graphs; accepted for publication in Raf. J. of Comp. \& Math's .

[6] F. Buckley and F. Harary (1990). Distance in Graphs. Addison-Wesley, Redwood.

[7] P. Dankelmann,W. Goddard, M.A. Henning and H.C. Swart; (1999). Generalized eccentricity, radius and diameter in graphs. Foundation for Research Development. (C) 1999 John Wiely \& Sons, Inc. Networks 34: 312-319.

[8] G. Chartrand and L. Lesniak (1986). Graphs and Digraphs, $2^{\text {nd }}$ ed., Wadsworth Inc., Belmont, California. 